\section{Perioperative FLOT superior to ECF/X}

Following the MAGIC trial, perioperative chemotherapy with epirubicin and cisplatin plus fluorouracil or capecitabine (ECF/X) has been the standard of care for localized gastric cancer. Now, findings from the FLOT4 trial challenge this paradigm by demonstrating the superiority of fluorouracil, leucovorin, oxaliplatin and docetaxel (FLOT) over $\mathrm{ECF} / \mathrm{X}$ as perioperative chemotherapy in locally advanced, resectable gastric cancer or gastro-oesophageal junction (GEJ) adenocarcinoma.

In the phase III portion of this trial, 716 patients with histologically confirmed, locally advanced ( $\geq \mathrm{cT} 2$ and/or $\mathrm{cN}+$ ), resectable disease were randomly allocated to have surgical resection and either three pre-operative and three postoperative cycles of ECF/X ( $n=360)$ or four pre-operative and four post-operative FLOT cycles $(n=356)$.

At a median follow-up duration of 43 months, overall survival (OS; the primary end point) was significantly longer in the FLOT group than in the ECF/X group (HR 0.77, 95\% CI 0.63-0.94; $P=0.012$; median OS 50 months versus 35 months). This OS benefit was consistent across subgroups stratified by baseline characteristics, including age, anatomical site, histology and clinical stage. Furthermore, compared with ECF/X, FLOT significantly improved the margin-free resection rate ( $78 \%$ versus $85 \% ; P=0.016$ ) and disease-free survival (HR $0.75,95 \% \mathrm{CI}$ 0.62-0.91; $P=0.0036$; median DFS 18 months versus 30 months).

Similar rates of serious (grade 3 or 4 ) treatment-related adverse events $(27 \%$ in both groups), toxic deaths ( $<1 \%$ in both groups) and hospitalization for toxicities (ECF/X 26\% versus FLOT 25\%) were reported between arms.

Overall, the findings of FLOT4 suggest that FLOT should replace ECF/X as the standard-of-care pre-operative regimen in patients with locally advanced, resectable gastric cancer or GEJ adenocarcinoma.

Conor A. Bradley

ORIGINAL ARTICLE Al-Batran, S.-E. et al. Perioperative chemotherapy with fluorouracil plus leucovorin, oxaliplatin, and docetaxel versus fluorouracil or capecitabine plus cisplatin and epirubicin for locally advanced, resectable gastric or gastro-oesophageal junction adenocarcinoma (FLOT4): a randomised, phase 2/3 trial. Lancet https:// doi.org/10.1016/S0140-6736(18)32557-1 (2019)

\title{
HDAC inhibitors tested in phase III trial
}

Epigenetic alterations can lead to changes in gene expression that underlie resistance to endocrine therapy, the standard-of-care treatment for patients with hormone receptor-positive $\left(\mathrm{HR}^{+}\right)$breast cancer. Now, the results of a phase III trial suggest that these patients could benefit from treatment with the histone deacetylase (HDAC) inhibitor tucidinostat.

In the ACE trial, postmenopausal women with $\mathrm{HR}^{+}{ }^{+} \mathrm{HER} 2^{-}$advanced-stage breast cancer resistant to endocrine therapy were randomly assigned to receive the aromatase inhibitor exemestane together with tucidinostat $(n=244)$ or placebo $(n=121)$. Investigator-assessed results (not shown herein) are consistent with those from independent evaluation. At 13.9 months, median progression-free survival (PFS) was longer in the tucidinostat group than in the placebo group: 9.2 months versus 3.8 months $(P=0.024)$. However, this trial was not sufficiently powered for the analysis of PFS (sample size and number of events).

The addition of tucidinostat to exemestane resulted in a higher objective response rate than that observed with placebo $(16 \%$ versus $7 \%$;
$P=0.024)$ and resulted in a higher percentage of patients deriving clinical benefit (defined in this trial as objective response or stable disease lasting $\geq 24$ weeks; $43 \%$ versus $31 \% ; P=0.02$ ). Grade 3-4 adverse events were reported in $75 \%$ of patients receiving tucidinostat plus exemestane and $16 \%$ of those receiving placebo plus exemestane.

Important aspects remain to be addressed: quality of life was not assessed and, most importantly, overall survival data were not available at the time of publication. Several HDAC inhibitors are already approved for the treatment of patients with haematological malignancies but, for this therapeutic class to be considered an option for patients with $\mathrm{HR}^{+}$ breast cancer resistant to endocrine therapy, mature results of this and other trials of HDAC inhibitors are needed.

Diana Romero

ORIGINAL ARTICLE liang, Z. et al. Tucidinostat plus exemestane for postmenopausal patients with advanced, hormone receptor-positive breast cancer (ACE): a randomised, double-blind, placebo-controlled, phase 3 trial. Lancet Oncol. https://doi.org/10.1016/ S1470-2045(19)30164-0 (2019)

\section{IMMUNOTHERAPY}

\section{Anti-BCMA CAR $T$ cells for MM}

Anti-CD19 chimeric antigen receptor (CAR)

$T$ cell therapies are efficacious and have been approved for the treatment of certain B cell leukaemias and lymphomas. CD19 is not typically expressed on the malignant plasma cells of multiple myeloma (MM), but a different $B$ cell marker, $B$ cell maturation antigen (BCMA), is expressed on these cells. Now, interim data from a phase I trial of a novel CAR $T$ cell product validate $B C M A$ as a therapeutic target in patients with MM.

The recently published data come from the first 33 patients treated with a single infusion of bb2121, comprising autologous T cells transduced with an anti-BCMA-4-1BB-CD3 $\zeta$ CAR construct, as part of the CRB-401 trial. All patients were heavily pretreated (median of 7 prior lines of therapy, range 3-23). Across a dose range of $50-800 \times 10^{6} \mathrm{CAR}$ $\mathrm{T}$ cells, the objective response rate (ORR) was $85 \%$ and the complete response (CR) rate was $45 \%$, with evidence of a dose-response relationship. Most patients $(n=19)$ received $450 \times 10^{6}$ CAR T cells; in this group, the ORR was $95 \%$ and was similar in subgroups stratified by BCMA expression on $<50 \%$ versus $\geq 50 \%$ of bone marrow plasma cells ( $100 \%$ and $91 \%$, respectively). Of note, 16 patients with a response were evaluated for minimal residual disease (MRD) and all tested negative at a sensitivity of $\leq 10^{-4}$.

At a median follow-up duration of 11.3 months (minimum of 6.2 months), the median progression-free survival was 11.8 months; $40 \%$ of responders had disease progression, with similar rates among those with a CR and/or a MRD-negative response. Thus, most responses are unlikely to persist.

Moreover, toxicities, although manageable, were common. Indeed, 97\% of patients had at least one grade $\geq 3$ adverse event $(A E)$, predominantly haematological AEs (for example, neutropenia in 85\%). Furthermore, the characteristic AEs of CAR T cell therapies were evident, with cytokine-release syndrome occurring in $76 \%$ of patients (grade 3 in $6 \%$ ) and neurological AEs in $42 \%$ (one of grade 4 ).

These data are promising but emphasize the value of ongoing translational research to improve not only the efficacy but also the safety of CAR T cell therapies.

David Killock

ORIGINAL ARTICLE Raje, N. et al. Anti-BCMA CAR T-cell therapy bb2121 in relapsed or refractory multiple myeloma. N. Engl.J. Med. 380, 1726-1737 (2019) 